\title{
Editorial
}

\section{TIMSS und PISA 2015}

\section{Was lernen wir aus den internationalen Schulleistungsstudien?}

\author{
Olaf Köller \\ Geschäftsführender Herausgeber
}

Im November (Trends in Mathematics and Science Study, TIMSS) und Dezember (Programme for International Student Assessment, PISA) des abgelaufenen Jahres 2016 wurden einmal mehr die Befunde internationaler Schulleistungsstudien der Öffentlichkeit vorgestellt. Deutschland beteiligt sich an TIMSS (vierte Jahrgangsstufe) und PISA (15-Jährige) mit national repräsentativen Stichproben. In TIMSS werden Naturwissenschaften und Mathematik getestet, in PISA Leseverstehen, Naturwissenschaften und Mathematik. An TIMSS in der Grundschule hat sich Deutschland zum dritten Mal beteiligt (nach 2007 und 2011), an PISA zum sechsten Mal (nach 2000, 2003, 2006, 2009 und 2012). In PISA 2015 lag der Schwerpunkt auf der Testung der Naturwissenschaften, in TIMSS wurden beide getesteten Domänen gleichermaßen gewichtet. Beide Studien haben den Anspruch, Informationen über die Leistungsfähigkeit der Bildungssysteme der Teilnehmerländer zu liefern, TIMSS in der Grundschule, PISA in der Sekundarstufe I. Zu beiden Studien sind zum Jahresende 2016 die nationalen Berichtsbände erschienen (Bos, Wendt, Köller \& Selter, 2016; Reiss, Sälzer, Schiepe-Tiska, Klieme \& Köller, 2016). Die Besonderheit bei PISA 2015 war, dass erstmalig alle Tests computerbasiert administriert wurden, in TIMSS werden nach wie vor Papier-und-Bleistift-Tests eingesetzt.

Beide Studien erlauben, deutsche Schülerinnen und Schüler in ihren Leistungen international zu vergleichen - in TIMSS mit den EU- und Organisation of Economic Co-operation and Development (OECD)-Staaten, in PISA nur mit den OECD-Staaten - und durch ihren wiederkehrenden Charakter (TIMSS alle vier Jahre, PISA alle drei Jahre) geben sie Möglichkeit, über den Kohortenvergleich sich Trends anzuschauen: Wie entwickeln sich die Leistungen der Viertklässlerinnen und Viertklässler seit 2007? Wie entwickeln sich die Leistungen der 15-Jährigen seit 2000? Während die Leistungen deutscher Schülerinnen und Schüler in TIMSS zwischen 2007 und 2011 stagnierten, sind die Leistungen in PISA zwischen 2000 und 2012 kontinuierlich leicht gestiegen, im Jahr 2012 lagen die Leistungen deutscher 15-Jähriger in allen drei getesteten Domänen erstmalig signifikant über den entsprechenden Mittelwerten aller OECD-Staaten. In allen drei Bereichen lagen die Leistungen der 15-Jährigen im Jahr 2012 signifikant höher als zu Beginn von PISA.

Was haben wir jetzt aus den neuen Runden im Jahr 2015 gelernt? In TIMSS stagnieren die mathematischen und naturwissenschaftlichen Leistungen der Viertklässlerinnen und Viertklässler erneut. Dieser Befund enttäuscht auf den ersten Blick, haben doch die 16 Länder der Bundesrepublik Deutschland in den letzten 15 Jahren große Anstrengungen unternommen, um die Förderung im Elementar- und Primarbereich zu verbessern. Sprachförderprogramme wurden auf den Weg gebracht, die Betreuung der unter Dreijährigen verbessert, der Ganztagesbetrieb in Grundschulen ausgebaut, Initiativen gestartet wie das Haus der kleinen Forscher und das Professionalisierungsprogramm SINUS an Grundschulen (vgl. Fischer, Rieck, Döring \& Köller, 2014). Das alles soll nicht gefruchtet haben? Man versteht die Befunde besser, wenn man sich die Komposition der in 2015 und in 2011 getesteten Kinder genauer anschaut. Dabei wird deutlich, dass der Anteil 
von Kindern mit Migrationshintergrund von rund $28 \%$ auf $32 \%$ gestiegen ist. Akzeptiert man, dass Kinder aus Familien mit Migrationshintergrund häufiger sozial, kulturell und ökonomisch benachteiligt sind (u. a. Tarelli, Schwippert \& Stubbe, 2012), so ist eigentlich bei einer Zunahme des Anteils dieser Kinder in der Gesamtkohorte mit einem Absinken der mittleren Leistungen zu rechnen. Dass dies in TIMSS nicht der Fall ist, mag dem erfolgreichen kompensatorischen Effekt der oben geschilderten Maßnahmen geschuldet sein. Es wird so deutlich, dass es dem System gelingt, Bildungsprozesse in einer sich verändernden Schülerschaft erfolgreich anzubahnen.

Noch spannender als die TIMSS-Befunde sind ohne Frage die PISA-2015-Ergebnisse, liegen hier doch immerhin Trendinformationen über einen Zeitraum von 15 Jahren vor. Nach zwölf Jahren steigender Leistungen der 15-Jährigen im Lesen, in der Mathematik und in den Naturwissenschaften markierte PISA 2015 einen Einschnitt: Stagnierende Leseleistungen, leicht sinkende mathematische Kompetenzen und deutlich abgesunkene naturwissenschaftliche Leistungen, und zwar in einer Größenordnung (17 Punkte), die sich weder mit einer veränderten Schülerschaft noch mit einer dramatischen Verschlechterung des Unterrichtsgeschehens in deutschen Schulen erklären lässt. Eine plausible Alternativerklärung, die von den PISA-Autorinnen und -Autoren in Deutschland angeboten und mit empirischer Evidenz unterfüttert wurde, bezieht sich auf die vielen Veränderungen, die in PISA 2015 gegenüber 2012 vorgenommen wurden. Anstelle von 13 Testversionen gab es jetzt 66, alte Aufgaben wurden von Papier-und-Bleistift- auf Computerformat umgestellt, neue dynamische naturwissenschaftliche Aufgaben wurden konstruiert und schließlich wurde auch noch das Skalierungsmodell (vom klassischen Raschmodell mit einem Schwierigkeitsparameter pro Item auf das 2-ParameterModell mit einem Schwierigkeits- und einem Diskriminationsparameter) geändert. Dass diese vielen Änderungen negative Effekte hatten, scheint abgesichert zu sein.
Was wir somit aus TIMSS und PISA lernen, ist, (a) dass das System erfolgreich auf ungünstigere Eingangsmerkmale der Schülerinnen reagieren kann, und (b) „If you want to measure change, don't change the measure“.

\section{Zum Schluss etwas in eigener Sache}

Die PEU geht in ihr 64. Jahr als Fachzeitschrift im Schnittbereich zwischen Entwicklungspsychologie und Pädagogischer Psychologie, immer mit einem starken Anwendungs- bzw. Praxisbezug. Die Zeitschrift fühlt sich nach wie vor dem Wissenschaftsmodus Theoria cum praxi (Wissenschaft zum Wohle der Gesellschaft), wie er vom großen Philosophen Gottfried Wilhelm Leibniz geprägt wurde, verpflichtet. Gute Praxis entsteht nicht erst aus guter Wissenschaft, wohl aber zeigt Wissenschaft, ob gefühlt gute Praxis auch nach ihrer wissenschaftlichen Überprüfung bestehen kann. Die PEU leistet somit nicht nur einen wichtigen Beitrag zur psychologischen Forschung, sondern hilft, evidenzbasiert Praxis weiter zu verbessern.

Die PEU hat nach wie vor sehr viele hochwertige Einreichungen, die angesichts des begrenzten Volumens der Hefte und des Einstreuens von Themenheften ein strenges Begutachtungssystem zur Folge haben. Im abgelaufenen Jahr 2015 war die Zahl der Einreichungen wieder so hoch, dass wir viele durchaus gute Manuskripte ablehnen mussten und nach wie vor bei einer Annahmequote von unter $40 \%$ liegen. Die Zahl der Manuskripte, die in der ersten Begutachtungsrunde akzeptiert werden, ist sehr gering (unter $20 \%$ ) und signalisiert die hohen Qualitätsstandards, die wir an Beiträge anlegen.

Hinsichtlich der Sichtbarkeit der PEUBeiträge ist festzustellen, dass der Impact-Factor im Journal Citation Report, der sich aktuell auf Zitationen im Jahre 2015 bezieht, leicht von 0,488 im Jahr 2014 auf 0,349 zurückgegangen ist. Dies mag Ansporn für die Herausgeberinnen und Herausgeber sein, die Attraktivität der Zeitschrift bei den Autorinnen und Autoren sowie Leserinnen und Lesern zu steigern. 


\section{Literatur}

Bos, W., Wendt, H., Köller, O. \& Selter, C. (Hrsg.). (2016). TIMSS 2015. Mathematische und naturwissenschaftliche Kompetenzen von Grundschulkindern in Deutschland im internationalen Vergleich. Münster: Waxmann.

Fischer, C., Rieck, K., Döring, B. \& Köller, O. (Hrsg.). (2014).Zusammenwirken - zusammen wirken. Unterrichtsentwicklung anstoßen, umsetzen, sichern. Seelze: Kallmeyer.
Reiss, K., Sälzer, C., Schiepe-Tiska, A., Klieme, E. \& Köller, O. (Hrsg.). (2016). PISA 2015: Eine Studie zwischen Kontinuität und Innovation. Münster: Waxman.

Tarelli, I., Schwippert, K. \& Stubbe, T. C. (2012). Mathematische und naturwissenschaftliche Kompetenzen von Schülerinnen und Schülern mit Migrationshintergrund. In W. Bos., H. Wendt, O. Köller \& C. Selter (Hrsg.), TIMSS 2011: Mathematische und naturwissenschaftliche Kompetenzen von Grundschulkindern in Deutschland im internationalen Vergleich (S. 247-267). Münster: Waxmann. 